4 Office of Population Censuses and Surveys. Hospital in-patient enquiry. HMSO, 1976

5 Office of Population Censuses and Surveys. Hospital in-patient enquiry, Table $R$ 1976, unpublished.

6 Office of Population Censuses and Surveys. Mortality statistics: cause. HMSO, 1976.

7 Vaughan DH. Allocation of additional consultant posts: critical review. Br Med F 1977 ;ii :843.

${ }^{8}$ London Health Planning Consortium. Acute hospital services in London. HMSO, 1979, 61 .
${ }^{9}$ London Health Planning Consortium Study Group on Methodology. Overview of the SGM methodology for projecting acute inpatient service requirements. Department of Health and Social Security, 1980, 5.

10 Barr A, Logan RFL. Policy alternatives for resource allocation. Lancet $1977 ; \mathrm{i}: 994$.

${ }^{11}$ Forster DP. Mortality and resource allocation. Lancet $1977 ; \mathrm{i}: 997$.

12 Knox EG. Principles of allocation of health care resources. 7 Epidemiol Community Health 1978;82:3.

(Accepted 15 October 1980)

\title{
Screening for congenital hypothyroidism in the Republic of Ireland
}

\author{
SYLVIA DOCKERAY，S F CAHALANE，MARY BRODY，CATHERINE MULLINS， M J CULLEN
}

\section{Summary and conclusions}

A national pilot study for detecting congenital hypothyroidism by radioimmunoassay of thyroid-stimulating hormone concentrations in dried blood was incorporated into the newborn screening programme in Ireland on 1 August 1979. The programme has been monitored by a steering committee and follows the guidelines set by the European Society of Paediatric Endocrinologists.

During the first 12 months 76224 infants were screened and 19 cases confirmed, giving an incidence of $1: 4012$. Fifty infants (0.07\%) were recalled for a serum sample, though most of the recalls $(31 ; 0.04 \%)$ occurred during the first three months, before the methodology had become established. No case was detected clinically. At recall only three of the 19 affected infants had obvious features of hypothyroidism, seven had mild features, and nine inconspicuous features. Organisation was directed at early diagnosis and treatment, the mean age at beginning treatment being 15 days.

These results confirm the efficacy of screening for congenital hypothyroidism and suggest that capital and running costs will be offset by savings in maintenance treatment of untreated patients. Screening does not, however, remove the need for continued vigilance, and clinicians should request thyroid-function tests in any suspected case.

\section{Introduction}

Like phenylketonuria, congenital hypothyroidism has apparently become a model condition for mass screening. Testing for congenital hypothyroidism was first introduced into a screening programme in Quebec in $1974,{ }^{1}$ and a recent leading article in the $B M \mathcal{F}$ concluded that further delays in implementing a national screening programme for the condition in Britain cannot be justified. ${ }^{2}$ Untreated, congenital hypothyroidism leads to mental

Children's Hospital, Dublin 1

SYLVIA DOCKERAY, MRCPI, DCH, acting consultant paediatrician S F CAHALANE, MD, FRCPI, consultant pathologist

MARY BRODY, BSC, biochemist

CATHERINE MULLINS, MSC, senior biochemist

St James's Hospital and the Federated Dublin Voluntary Hospitals, Dublin

M J CULLEN, $\mathrm{MB}, \mathrm{BCH}$, consultant endocrinologist and physical retardation, and the disease is rarely diagnosed clinically before irreversible nervous system damage is established. Some three and a half million babies have now been screened for hypothyroidism, the worldwide case-detection rate being 1:4435 (Western Europe 1:3976, North America 1:4299, Australia 1:5817, Japan 1:6884). ${ }^{3}$ Hulse et al reported an incidence of $1: 3363$ among newborn infants in North London and adjoining counties. ${ }^{4}$ Detection of congenital hypothyroidism by thyroid-stimulating hormone (TSH) assay on dried-blood filter-paper specimens is a sensitive and practical procedure with a low recall rate. Confirmatory assays are in the repertoire of all endocrine laboratories and within the competence of general clinical laboratories experienced in radioimmunoassay. The efficacy of treatment-namely, simple oral replacement-has been proved, and mass screening and early institution of treatment will apparently be an economic asset to the community compared with maintaining untreated patients. ${ }^{5}$

A national newborn blood phenylketonuria screening service for Ireland was established in the pathology department of the Children's Hospital in 1966, the Guthrie microbiological inhibition assay procedure being used on dried blood collected by heel stab on the fourth or fifth day of life. ${ }^{6}$ Similar tests have been added at intervals for other inherited metabolic errors, and the entire newborn population of Ireland is now screened for phenylketonuria, homocystinuria, galactosaemia, tyrosinaemia, maple syrup urine disease, and, since August 1979, hypothyroidism. The additional metabolite tests added little to the cost of testing for phenylketonuria, but capital and running costs were increased when screening for congenital hypothyroidism was included.

We describe the incorporation of screening for hypothyroidism into the national newborn dried-blood screening programme in the Republic of Ireland and present the results of a 12-month pilot study.

\section{Materials and methods}

The programme was funded by the Department of Health, and the 12 -month pilot study was begun on 1 August 1979. The screening procedure consisted in estimating by radioimmunoassay thyroidstimulating hormone concentrations in heel-stab samples of dried blood. A steering committee was set up, comprising a medical representative from the Department of Health, paediatricians from centres throughout Ireland, a consultant endocrinologist and consultant radiologist, and the laboratory director and clinical biochemists concerned. One of us (SD) acted as secretary and clinical co-ordinator for the study. The committee met monthly to review laboratory results and case records and discuss protocol in relation to developments.

Collection of blood samples-Heel-stab samples are collected on filter 
paper before discharge from the maternity unit, the advice being that this should be done on the fourth day of life. Specimens are collected from all newborn in the Republic of Ireland. Screening for hypothyroidism necessitated adding a further circle for blood on the collecting card to prevent overspotting; for although this and consequent supersaturation of the filter paper is acceptable for the microbiological assays, it results in misleadingly high TSH concentrations and hence a high recall rate.

Laboratory organisation-The organisation of the screening programme has been described. ${ }^{7}$ The screening laboratory is sited in the hospital pathology department and supervised by SFC. Thyroid hormone assays had not been carried out before, though the laboratory was experienced in radioimmunoassay procedures. A special small laboratory was established immediately adjacent to the main laboratory. A basic-grade biochemist was appointed but screening staff were not increased; other biochemistry personnel were trained in the procedure to serve as relief staff. Discs are hand punched from blood specimens into tubes before being transferred in racks to the radioimmunoassay laboratory. Some 300 specimens are assayed daily during Monday to Friday.

Radioimmunoassay-A commercial solid-phase kit method (Phadebas dry-spot TSH test, Pharmacia Diagnostics, Uppsala) is used on a 4.25 $\mathrm{mm}$ disc punched from the filter paper. The discs are eluted for four hours with $0.05 \mathrm{ml}$ Sephadex-anti-TSH complex and incubated overnight after the addition of $0.1 \mathrm{ml}{ }^{125} \mathrm{I}$-labelled TSH. Dry-blood standards and controls are provided in the kits, and samples from known cases of primary hypothyroidism are included in each run to check for drift. Sample identification is achieved throughout by means of a rack system. The racks are designed for use in the dispenser (LKB 2071 sample processor) and gamma counter (LKB 1270 Rack Gamma carefully monitored. As a result of the report by J F Connolly et al, however (eighth international thyroid congress, Sydney, February 1980), we now offer a conventional pertechnetate $\left({ }^{9} \mathrm{~m} \mathrm{mc}\right)$ thyroid scan in all new cases. The technique is simple, takes under 30 minutes, and has delayed replacement therapy by at most only one day. Pertechnetate is readily available, unlike ${ }^{125} \mathrm{I}$. A pinhole collimator and gammacamera are required, and ${ }^{99 \mathrm{~m}} \mathrm{Tc}$ is given as an intravenous bolus of $200 \mu \mathrm{Ci}$. Irradiation to the thyroid is about $42 \mathrm{mR}$, compared with 200-500 mR during one chest $x$-ray examination. The scans have been carried out in the department of nuclear medicine at St James's Hospital, Dublin, the infants being admitted overnight to the Children's Hospital.

\section{Results}

During the pilot study 76224 infants were screened; $50(0.07 \%)$ were recalled for a serum sample and 19 cases of congenital hypothyroidism were confirmed, giving an incidence of 1:4012. In the 19 confirmed cases the first blood spot was collected between days 3 and 11. Initial testing was conducted between days 5 and 15 and (in 18 cases) confirmatory serum testing between the 10th and 23rd days (in case 3 testing was delayed by administrative problems). Table II shows the distribution of dried blood-spot TSH concentrations in the series. Only 447 infants $(0.06 \%)$ had values exceeding $20 \mathrm{mU} / \mathrm{l}$ and $33(0.04 \%)$ values exceeding $40 \mathrm{mU} / 1$ on initial assay. Table III gives the TSH and thyroxine concentrations in the confirmed cases.

The recall rate $(0.07 \%)$ was remarkably low. Nevertheless, the rate was higher in the early months $(0.16 \%)$, probably owing to technical problems and faults in the collection technique-for example, over-

TABLE I-Protocol for diagnosis and management

\begin{tabular}{lcc}
\hline Confirmation of diagnosis & Timing & Procedures \\
\hline $\begin{array}{l}\text { Monitoring } \\
\text { Re-evaluation of diagnosis }\end{array}$ Follow-up & $\begin{array}{l}\text { Weekly then every 1-3 months } \\
\text { 1 year }\end{array}$ & $\begin{array}{c}\text { Serum TSH and thyroxine estimation; full clinical history and examination, including length } \\
\text { weight, and skull circumference recorded on study questionnaire; radiography of knee, } \\
\text { clinical photograph, pertechnetate thyroid scan; start treatment with thyroxine 50 } \mu g \text { daily } \\
\text { Fast weekly measurement of TSH; thyroxine-estimation service available for monitoring } \\
\text { treatment } \\
\text { After short withdrawal of treatment, measurement of serum thyroxine, TSH, triiodothyronine, } \\
\text { free thyroxine, and thyroxine-binding globulin; thyroid scanning (if not done at time } \\
\text { of diagnosis) } \\
\text { Height, weight, head circumference, radiography for bone age } \\
\text { Standardised developmental testing }\end{array}$ \\
\hline
\end{tabular}

TABLE II-Distribution of dried blood-spot TSH concentrations in 76224 newborns

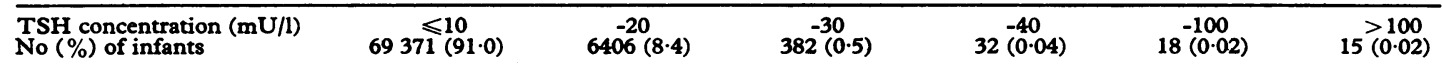

II). Carrier adaptors are used in the Damon/IEC centrifuge, so that 16 racks with 160 tubes may be centrifuged simultaneously. The LKB 1270 Rack Gamma II incorporates a teleprinter controlled by microprocessor. Automatic programming provides standard-curve fitting with spline function (counts/min versus log concentration) and calculation of unknowns.

Recall policy-Blood-spot TSH concentrations up to $20 \mathrm{mU} / 1$ are regarded as normal. If the value is $21-40 \mathrm{mU} / 1$, however, the test is repeated on the original specimen, and if the concentration is still raised the infant is recalled for a serum sample. When a value exceeds $40 \mathrm{mU} / 1$ a serum sample and a further filter-paper specimen are immediately requested by telephone and blood from the original card reprocessed. To confirm the diagnosis $1 \mathrm{ml}$ serum is requested for measurement of thyroxine and TSH concentrations. The samples are processed as an emergency in the central biochemistry laboratory of the Federated Dublin Voluntary Hospitals, where the duration of the procedure has been reduced to four hours. When the diagnosis is confirmed the clinical co-ordinator contacts the local consultant paediatrician to arrange management according to the protocol.

Protocol-Guidelines for management were drawn up by the steering committee and accorded with the recommendations of the European Society of Paediatric Endocrinologists (table I). The initial intention was to defer ${ }^{125} \mathrm{I}$ thyroid scan until re-evaluation at 12 months to prevent delay in beginning treatment and avoid unnecessary irradiation in the newborn period. Since positive identification of transient cases would not therefore be possible until 1 year of age, treatment was saturation of the dried blood spot. During the last nine months only 19 babies $(0.03 \%)$ were recalled for a serum sample, and 14 of these were confirmed as having congenital hypothyroidism. Of the infants with confirmed disease, five (cases $7,12,16,17$, and 19) had dried bloodspot TSH concentrations below $80 \mathrm{mU} / 1$. Three of these (cases 7, 12 , and 17), including an infant born at 31 weeks of gestation (case 17), had normal or borderline serum thyroxine concentrations and were thought to be transient cases.

One case escaped detection by screening and was diagnosed clinically at 7 weeks of age. The initial dried blood spot, collected on the fourth day, had yielded a normal TSH concentration, and this was confirmed both by us and a reference laboratory after the clinical diagnosis had been made.

During the last four months of the study pertechnetate thyroid scan was included in the protocol to confirm the diagnosis. Ten infants were scanned, resulting in diagnoses of agenesis in two, ectopic thyroid in six, hypoplastic gland in one, and unilateral thyroid gland with normal uptake in one. This last infant (case 12), who presented as a clinically normal child with raised TSH and normal thyroxine concentrations, was regarded as an example of transient hyperthyrotrophinaemia and required long-term follow-up only.

Congenital hypothyroidism had apparently not been suspected clinically in any of the affected infants before recall. Nevertheless, at the time of recall three infants (cases 6, 9, and 13) had obvious clinical features (serum thyroxine concentrations $<10,60$, and $28 \mathrm{nmol} / \mathrm{l}$ $(<0 \cdot 8,4 \cdot 7$, and $2 \cdot 2 \mu \mathrm{g} / 100 \mathrm{ml})$ respectively), including coarse facies, 
TABLE III-Results in 19 confirmed cases of congenital hypothyroidism

\begin{tabular}{|c|c|c|c|c|c|c|c|}
\hline \multirow{2}{*}{ Case No } & \multirow{2}{*}{$\begin{array}{l}\text { TSH concentration } \\
\text { in dried blood } \\
\text { spot }(\mathrm{mU} / \mathrm{l})\end{array}$} & \multicolumn{2}{|c|}{ Confirmatory serum concentrations } & \multicolumn{3}{|c|}{ Age (days) } & \multirow{2}{*}{$\begin{array}{c}\text { Collection- } \\
\text { confirmation } \\
\text { interval (days) }\end{array}$} \\
\hline & & $\mathrm{TSH} \mathrm{mU} / \mathrm{l}$ & Thyroxine (nmol/1) & Collection & Testing & Confirmation & \\
\hline $\begin{array}{r}1 \\
2 \\
3 \\
4 \\
5 \\
6 \\
7 \\
8 \\
9 \\
10 \\
11 \\
12 \\
13 \\
14 \\
15 \\
16 \\
18 \\
19\end{array}$ & $\begin{array}{r}126 \\
128 \\
91 \\
126 \\
202 \\
140 \\
31 \\
117 \\
90 \\
84 \\
145 \\
31 \\
100 \\
85 \\
88 \\
39 \\
29 \\
122 \\
69\end{array}$ & $\begin{array}{r}908 \\
181 \\
72 \\
202 \\
>455 \\
375 \\
68 \\
>200 \\
190 \\
>200 \\
>200 \\
61 \\
171 \\
>171 \\
>182 \\
106 \\
68 \\
>150 \\
>150\end{array}$ & $\begin{array}{r}68 \\
101 \\
148 \\
47 \\
28 \\
<10 \\
190 \\
88 \\
60 \\
89 \\
55 \\
171 \\
28 \\
55 \\
46 \\
145 \\
128 \\
75 \\
60\end{array}$ & $\begin{array}{r}7 \\
5 \\
4 \\
4 \\
6 \\
4 \\
4 \\
3 \\
3 \\
3 \\
3 \\
4 \\
5 \\
5 \\
4 \\
4 \\
11 \\
5 \\
4\end{array}$ & $\begin{array}{r}99 \\
6 \\
10 \\
6 \\
13 \\
9 \\
10 \\
10 \\
5 \\
6 \\
11 \\
14 \\
10 \\
9 \\
12 \\
14 \\
15 \\
7 \\
8\end{array}$ & $\begin{array}{ll}15 \\
16 \\
29 * \\
13 \\
16 \\
15 \\
18 \\
13 \\
10 \\
11 \\
17 \\
21 \\
14 \\
12 \\
16 \\
21 \\
23 \\
11 \\
14\end{array}$ & $\begin{array}{r}8 \\
11 \\
25 \\
9 \\
10 \\
11 \\
14 \\
10 \\
7 \\
8 \\
14 \\
17 \\
9 \\
7 \\
12 \\
17 \\
12 \\
6 \\
10\end{array}$ \\
\hline
\end{tabular}

* Testing delayed by administrative difficulties.

Conversion: SI to traditional units-Serum thyroxine: $1 \mathrm{nmol} / 1 \approx 0.08 \mu \mathrm{g} / 100 \mathrm{ml}$. (Normal range in neonates in reference laboratory $150-300 \mathrm{nmol} / 1$ (11.7-23.3 $\mu \mathrm{g} / 100 \mathrm{ml}$.$) )$

enlarged tongue, enlarged posterior fontanelle, hypotonicity, constipation, feeding problems, and subnormal temperature. Milder but none the less characteristic features such as prominent tongue and small umbilical hernia were noted in seven (cases $5,8,10,11,14,15$, and 18). The remaining nine infants were clinically normal, including three diagnosed as probable cases of transient hypothyroidism, one of whom was preterm. Thus only three of the 19 confirmed cases of hypothyroidism would be likely to have had an early clinical diagnosis. The incidence of transient cases will be reported once the infants have reached 1 year of age.

On confirmation of the diagnosis all but three infants (cases 3, 7, and 12) began treatment on the 11 th to 23 rd days of life (mean 15 days). Of the exceptions, in case 3 confirmation was delayed until day 29 due to difficulty in follow-up, and case 7 , who was asymptomatic but had a persistent mild increase in TSH concentration, began treatment at 10 weeks of age when a borderline serum thyroxine concentration was recorded: a pertechnetate scan will be carried out on re-evaluation at 1 year of age. The third infant (case 12) is referred to above. Distance from the screening laboratory did not influence the timing of diagnosis; the infant farthest removed (case 10) had treatment initiated on the 13th day, investigations having included a pertechnetate scan. Management was simplified by a weekly. biochemical monitoring service for all infants detected by the programme.

Costing-The more expensive items of equipment, including automatic sample processor, gamma counter, and refrigerated centrifuge, were acquired by a lease agreement for the pilot study with an option to purchase thereafter. The cost of this arrangement plus other equipment, furnishing, reagents, and staff was $68 \mathrm{p}$ per neonate tested. Administrative overheads, such as specimen collection, postage, and telephone, were met under the national neonatal screening programme.

\section{Discussion}

Screening programmes for congenital hypothyroidism have been established in many centres throughout the world but few are organised nationally. Because of the population size and yearly birth rate, Ireland lends itself to the organisation of a central screening laboratory, and it is within this framework that screening for congenital hypothyroidism by TSH radioimmunoassay has been incorporated. The clinical management of confirmed cases is straightforward, and the policy has been for the infant to remain under the care of the local paediatrician. Before the pilot study began all paediatricians were circulated with details of the screening programme and protocol, and we have received their full co-operation. The steering committee has provided an extremely helpful channel of communication between the parties concerned in the study.

The merits of other procedures for screening for congenital hypothyroidism-for example, cord blood or first-week driedblood-spot assay of thyroxine with supplemental TSH estimation, or TSH alone-have been discussed. ${ }^{13}$ Radioimmunoassay of TSH in first-week dried-blood spots was chosen for the pilot study and was effectively incorporated into the existing screening programme.

The aim of the programme is to prevent the sequelae of congenital hypothyroidism, and our priority has been to provide early diagnosis and begin treatment at the earliest opportunity. Wolter et $a l,{ }^{8}$ who studied the neuropsychological sequelae of treated thyroid dysgenesis, tried to define the time of onset and duration of hypothyroidism for each child from bone age. They showed that by treating prenatal hypothyroidism before 1 month of age the IQ was reversed to normal, but minor neuropsychological defects remained. These were also present in infants with postnatal onset of hypothyroidism, who were all shown to have a normal IQ. Their earliest-treated child was 18 days old, and they commented that better results may be expected from earlier treatment, which should be possible for children detected by a well-organised screening programme.

The mean age at beginning treatment in this study (15 days) was half that in the study reported by Hulse et al. ${ }^{4}$ In the Irish programme the diagnosis is accelerated by the earlier age at heelstab sampling (4-6 days), by carrying out assays five days a week, and by recalling the infant for a serum sample when the bloodspot TSH value exceeds $40 \mathrm{mU} / 1$, such samples being processed as an emergency. The low recall rate justifies this procedure. The only unavoidable delay is postal, which occurs occasionally in the delivery of blood cards. The effectiveness of early testing and rapid follow-up in the Irish screening programme was referred to by Starfield and Holtsman, who compared the effectiveness of screening for phenylketonuria in the USA, Britain, and Ireland. ${ }^{9}$

With the low recall rate and subsequent low false-positive rate emotional stress to parents of normal children is minimal. Interestingly, of the 19 newly detected cases, 17 were born during April to September. The geographical distribution of detected cases was apparently fairly even. One case was missed by screening, which emphasises the need for continuing vigilance; hence clinicians should not hesitate to request thyroid function tests in suspected cases, even when comprehensive screening is in operation.

The pilot programme for congenital hypothyroidism is continuing as a permanent component of screening in Ireland. The effectiveness of screening and early treatment will be apparent only when long-term follow-up of detected cases has been completed. As in other programmes the results of early developmental testing are encouraging.

Members of the steering committee are as follows: Professor O C Ward (chairman), Dublin; Miss M Brody, Dublin; Dr S F Cahalane, Dublin; Dr M Cullen, Dublin; Dr G Cussen, Cork; Dr S Dockeray, Dublin; Dr T Fitzgerald, Department of Health, Dublin; Dr E Griffin, Dublin; Dr D Lillis, Galway; Dr J McCarthy, Dublin; Dr T Matthews, Dublin; Mrs C Mullins, Dublin; Dr N O'Brien, Dublin; 
Dr J O'Sullivan, Drogheda; Dr J P R Rees, Dublin; Dr M Curtin, Limerick.

We acknowledge the help and guidance of the steering committez, and also Dr P Freyne for p zrtech retate scans; Adani Cranny and staff of the endocrinology section of the central biochemistry laboratories of the Federated Dublin Hospitals; and Mrs Sheila Flynn and the screening laboratory staff Fiona McSweeney, D Liddell, and Denise Horgan, of the Children's Hospital biochemistry laboratory. The staff of Pharmacia (Great Britain and Sweden) have been most helpful Finally, our best thanks go to Mrs Anna Power and Miss Maria Brooks for secretarial work.

\section{References}

1 Dussault JH, Coulombe P, Laberge C, Letarte J, Guyda H, Khoury K. Preliminary report on a mass screening for neonatal hypothyroidism. f Pediatr $1979 ; 85: 670-4$.

2 Anonymous. Screening for congenital hypothyroidism. Br Med $\mathcal{f} 1980$; $281: 1-2$.
${ }^{3}$ Fisher DA. Status of neonatal hypothyroidism screening: report from Quebec international conference on neonatal thyroid screening. Stockist JR, Nagataki S, eds. In: Proceedings of the eighth international thyroid congress, Sydney, Australia, 1980. Canberra: Australian Academy of Science, 1980:1-7.

4 Hulse JA, Grant DB, Clayton BE, et al. Population screening for congenital hypothyroidism. Br Med $\mathcal{F} 1980 ; 280: 675-8$.

5 Smith P, Morris A. Assessment of a programme to screen the newborn for congenital hypothyroidism. Community Medicine 1979;1:14-22.

6 Cahalane SF. Phenylketonuria: mass screening for newborns in Ireland. Arch Dis Child 1968;43:141-4.

${ }^{7}$ Cahalane SF. Organisation of screening laboratories. In: Bickel $\mathbf{H}_{\text {, }}$ Guthrie R, Hammersen G, eds. Neonatal screening for inborn errors of metabolism. Berlin: Springer, 1980:287-8.

8 Wolter R, Noël P, De Cock M, et al. Neuropsychological study in treated thyroid dysgenesis. Acta Paediatr Scand 1979;277, suppl:41-6.

9 Starfield B, Holtsman NA. A comparison of effectiveness of screening for phenylketonuria in the United States, United Kingdom and Ireland. $N$ Engl F Med 1975 ;293:118-21.

(Accepted 25 September 1980)

\title{
How long does it take to become fit?
}

\author{
JOHN PEARN
}

\section{Summary and conclusions}

To become fit an individual must generate optimal muscle strength and must develop cardiopulmonary reserve, or stamina. Physical fitness programmes require motivation, a graded series of appropriately designed exercises, and scientific surveillance. Motivation and efficiency in fitness programmes depends on early positive feedback to participants, confirming that stamina and strength are developing.

A practical field experiment was performed to determine the minimum time that healthy young adults require to reach an initial plateau in objective measures of fitness. Fifty male university undergraduates were studied during an annual volunteer military training camp. Thirty had volunteered to take part in the fitness programme; the remaining 20 had initially rejected the offer but underwent the programme as part of their military training and acted as unmotivated controls. All the subjects became fit within 14 days of starting training, with objective improvement in both absolute strength and pulse recovery times.

Non-motivated individuals, training with motivated individuals for 20 minutes each day, can therefore achieve levels of fitness indistinguishable from those of healthy highly motivated subjects. Fitness programmes must be carefully supervised, however, with medical examinations for those about to undergo vigorous exercise.

\section{Introduction}

Current interest in physical fitness programmes is high. To become fit an individual must develop both optimal muscle strength and cardiopulmonary reserve or stamina. The

2 Field Hospital, Brisbane, Australia

JOHN PEARN, MD, lieutenant-colonel, RAAMC generation of, and tests for, these different facets of fitness are quite distinct but complementary. ${ }^{1}$ To achieve these two prerequisites of fitness, an out-of-condition sedentary individual must have (a) motivation, (b) a suitable training and exercise environment, ${ }^{2}$ and $(c)$ an acceptance of some inevitable physical discomfort.

Persistence with a programme of fitness training depends largely on the certainty that success will be possible and on the presence of signs that improvement is occurring rapidly. For all but children and healthy young adults, exercise challenge should be gradual and unobtrusive ${ }^{3}$ and medically supervised. Analysis of motivational factors leading to participation in vigorous physical training has shown that a desire to keep fit and to achieve a subjective feeling of well being is the most important. $^{35}$ It is known, however, that positive attitudes towards physical education are not necessarily correlated with high scores of physical fitness. ${ }^{6}$

In the case of adolescents and young adults, motivation is quickly lost if results are not achieved. "How long does it take to get fit ?" is thus an important question that the doctor is asked whenever he recommends that an individual start a training programme. I report here the results of a study to answer this question in subjects of both high and low initial motivation.

\section{Methods}

Background-This study was undertaken during routine training in an annual army camp (Army Reserve) of 16 days' duration. Test subjects comprised 50 healthy male students aged 18-24 years.

Physical fitness programme-A standard 10-station training circuit ${ }^{7}$ was established. Each station consisted of a standard physical task (Harvard step test, "chin-up" horizontal bar, body-press site, etc) Subjects set their own initial task baselines in standard fashion. ${ }^{4}$ The fitness programme then consisted of three sequential circuits of the 10-station course, at maximum speed against a stopwatch. Each individual undertook the training session twice daily for 14 consecutive days, under close supervision. Course times varied from eight to 14 minutes (see fig 1). Unlike other combat conditioning courses, subjects in this study were not involved in other strenuous physical activities during the duration of the tests. Normal warm-ups were 